\title{
FURTHER RESULTS ON ODD HARMONIOUS GRAPHS
}

\author{
M. E. Abdel-Aal ${ }^{1}$, M. A. Seoud ${ }^{2}$ \\ ${ }^{1}$ Department of Mathematics, Faculty of Science, \\ Banha University, Banha 13518, Egypt \\ ${ }^{2}$ Department of Mathematics, Faculty of Science, \\ Ain Shams University, Abbassia, Cairo, Egypt.
}

\begin{abstract}
In [1] Abdel-Aal has introduced the notions of m-shadow graphs and $n$-splitting graphs, for all $m, n \geq 1$. In this paper, we prove that, the m-shadow graphs for paths and complete bipartite graphs are odd harmonious graphs for all $m \geq 1$. Also, we prove the $n$-splitting graphs for paths, stars and symmetric product between paths and null graphs are odd harmonious graphs for all $n \geq 1$. In addition, we present some examples to illustrate the proposed theories. Moreover, we show that some families of graphs admit odd harmonious libeling.
\end{abstract}

\section{KEYWORDS}

Odd harmonious labeling, m-shadow graph, m-splitting graph.

MATHEMATICS SUBJECT CLASSIFICATION : 05C78, 05C76, 05C99.

\section{INTRODUCTION}

We begin with simple, finite, connected and undirected graph $G=(V, E)$ with p vertices and $\mathrm{q}$ edges. For all other standard terminology and notions we follow Harary[4].

Harmonious graphs naturally arose in the study by Graham and Sloane [3].

They defined a graph $G$ with $q$ edges to be harmonious if there is an injection $f$ from the vertices of $G$ to the group of integers modulo $q$ such that when each edge $x y$ is assigned the label $f(x)+$ $f(y)(\bmod q)$, the resulting edge labels are distinct.

A graph $G$ is said to be odd harmonious if there exists an injection $f: V(G) \rightarrow\{0,1,2, \ldots, 2 q-1\}$ such that the induced function $f^{*}: E(G) \rightarrow\{1,3, \ldots, 2 q-1\}$ defined by $f^{*}(u v)=f(u)+f(v)$ is a bijection. Then $f$ is said to be an odd harmonious labeling of $G$ [5]. A graph which has odd harmonious labeling is called odd harmonious graph.

For a dynamic survey of various graph labeling problems we refer to Gallian [2].

DOI : 10.5121/jgraphoc.2016.8401 
International Journal on Applications of Graph Theory in Wireless Ad hoc Networks and Sensor Networks

(GRAPH-HOC) Vol.8, No.3/4, December 2016

Liang and Bai [5] obtained the necessary conditions for the existence of odd harmonious labeling of a graph. They proved that if $G$ is an odd harmonious graph, then $G$ is a bipartite graph. Also they claim that if a $(p, q)$ - graph $G$ is odd harmonious, then $2 \sqrt{q} \leq p \leq 2 q-1$, but this is not always correct. Take $P_{2}$ as a counter example.

In [1] Abdel-Aal has introduced an extension for shadow graphs and splitting graphs. Namely, for any integers $m \geq 1$, the $m$-shadow graph denoted by $D_{m}(G)$ and the $m$ - splitting graph denoted by $\operatorname{Spl}_{m}(G)$ are defined as follows:

Definition 1.1. [1] The $m$-shadow graph $D_{m}(G)$ of a connected graph $G$ is constructed by taking $m$-copies of $G$, say $G_{1}, G_{2}, G_{3}, \ldots, G_{m}$, then join each vertex $u$ in $G_{i}$ to the neighbors of the corresponding vertex $v$ in $G_{j}, 1 \leq i, j \leq m$.

Definition 1.2. [1] The $m$ - splitting graph $\operatorname{Spl}_{m}(G)$ of a graph $G$ is obtained by adding to each vertex $v$ of $G$ new $m$ vertices, say $v^{1}, v^{2}, v^{3}, \ldots, v^{m}$, such that $v^{i}, 1 \leq i \leq m$ is adjacent to every vertex that is adjacent to $v$ in $G$.

Vaidya and Shah [6] proved that the shadow graphs of the path $P_{n}$ and the star $K_{1, n}$ are odd harmonious. Further they prove that the splitting graphs of the path $P_{n}$ and the star $K_{1, n}$ admit odd harmonious labeling.

In this paper, we initiate the study by proving that $D_{m}\left(P_{n}\right)$, for each $m, n \geq 1$ is odd harmonious, and we show that $D_{m}\left(K_{r, s}\right)$ for each $m, r, s \geq 1$ admits an odd harmonious labeling. Further, we prove that, the following graphs $\operatorname{Spl}_{m}\left(P_{n}\right), \operatorname{Spl}_{m}\left(K_{1, n}\right), P_{n} \oplus \overline{K_{m}}, \operatorname{Spl}_{m}\left(P_{n} \oplus \overline{K_{2}}\right), \operatorname{Spl}_{m}\left(P_{2} \wedge S_{n}\right)$, $\operatorname{Spl}\left(K_{m, n}\right)$ are odd harmonious.

\section{MAin RESUltS}

\section{Theorem 2.1.}

All connected graphs of order $\leq 6$ are not odd harmonious, except the following 25 graphs:

(i) $C_{4}, K_{2,3}, K_{2,4}, K_{3,3}$,

(ii) all non-isomorphic trees of order $\leq 6$,

(iii) the following graphs of order $\leq 6$ 
International Journal on Applications of Graph Theory in Wireless Ad hoc Networks and Sensor Networks

(GRAPH-HOC) Vol.8, No.3/4, December 2016

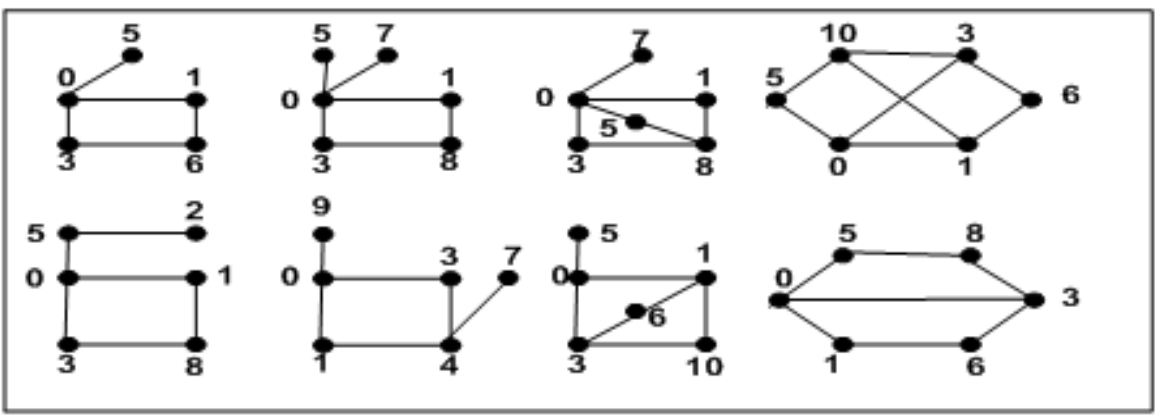

Figure (1)

Proof.

The graphs in (i) are odd harmonious by theorems due to Liang and Bai [5]; note that the graphs in case (ii) are exactly 13 non-isomorphic trees of order $\leq 6$, and they and the graphs in case (iii) are shown to be odd harmonious by giving specific odd harmonious labeling assigned to the vertices of each graph as indicated in Figure (1) and Figure (2). According to Harary [4], the remaining (118) connected graphs of order $\leq 6$, are not odd harmonious by the theorem: "Every graph with an odd cycle is not odd harmonious" which is also due to Liang and Bai [5].

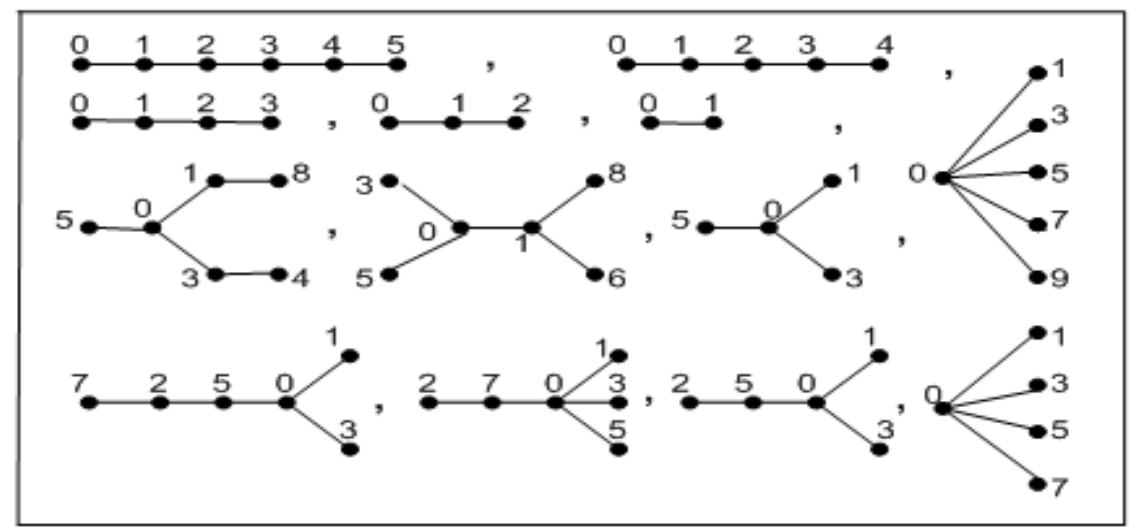

Figure (2)

Theorem 2.2. $D_{m}\left(P_{n}\right)$ is an odd harmonious graph for all $m, n \geq 2$.

Proof. Consider $m$-copies of $P_{n}$. Let $u_{1}^{j}, u_{2}^{j}, u_{3}^{j}, \ldots, u_{n}^{j}$ be the vertices of the $j^{\text {th }}$-copy of $P_{n}$. Let $G$ be the graph $D_{m}\left(P_{n}\right)$. Then $|V(G)|=m n$ and $|E(G)|=m^{2}(n-1)$. We define $f: V(G) \rightarrow\{0,1$, $\left.2, \ldots, 2 m^{2}(n-1)-1\right\}$ as follows:

$$
f\left(u_{i}^{j}\right)=\left\{\begin{array}{lll}
m^{2}(i-1)+2 m(j-1) & i=1,3,5, \ldots, n \text { or } n-1, & 1 \leq j \leq m \\
m^{2}(i-2)+2 j-1 & i=2,4,6, \ldots, n-1 \text { or } n, & 1 \leq j \leq m
\end{array}\right.
$$

It follows that $f$ is an odd harmonious labeling for $D_{m}\left(P_{n}\right)$. Hence $D_{m}\left(P_{n}\right)$ is an odd harmonious graph for each $m, n \geq 1$. 
International Journal on Applications of Graph Theory in Wireless Ad hoc Networks and Sensor Networks (GRAPH-HOC) Vol.8, No.3/4, December 2016

Example 2.3. An odd harmonious labeling of the graph $D_{4}\left(P_{7}\right)$ is shown in Figure (3).

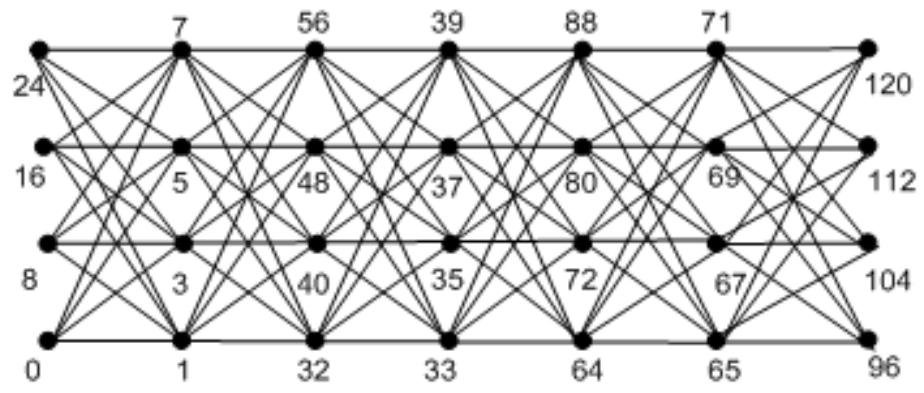

Figure (3): The graph $D_{4}\left(P_{7}\right)$ with its odd harmonious labeling

Theorem 2.4. $D_{m}\left(K_{r, s}\right)$ is an odd harmonious graph for all $m, r, s \geq 1$.

Proof. Consider $m$-copies of $K_{r, s}$. Let $u_{1}^{j}, u_{2}^{j}, u_{3}^{j}, \ldots, u_{r}^{j}$ and $v_{1}^{j}, v_{2}^{j}, v_{3}^{j}, \ldots, v_{s}^{j}$ be the vertices of the $j^{\text {th }}$-copy of $K_{r, s}$. Let $G$ be the graph $D_{m}\left(K_{r, s}\right)$. Then $|V(G)|=m(r+s)$ and $|E(G)|=m^{2} r s$. We define

$$
f: V(G) \rightarrow\left\{0,1,2, \ldots, 2 m^{2} r s-1\right\}
$$

as follows:

$$
\begin{array}{ll}
f\left(u_{i}^{j}\right)=2(i-1)+2 r(j-1), & 1 \leq i \leq r, 1 \leq j \leq m . \\
f\left(v_{i}^{j}\right)=1+2 m r(i-1)+2 m r s(j-1), & 1 \leq i \leq s, 1 \leq j \leq m .
\end{array}
$$

It follows that $f$ is an odd harmonious labeling for $D_{m}\left(K_{r, s}\right)$. Hence $D_{m}\left(K_{r, s}\right)$ is an odd harmonious graph for each $m, r, s \geq 1$.

Example 2.5. An odd harmonious labeling of the graph $D_{3}\left(K_{3,4}\right)$ is shown in Figure (4).

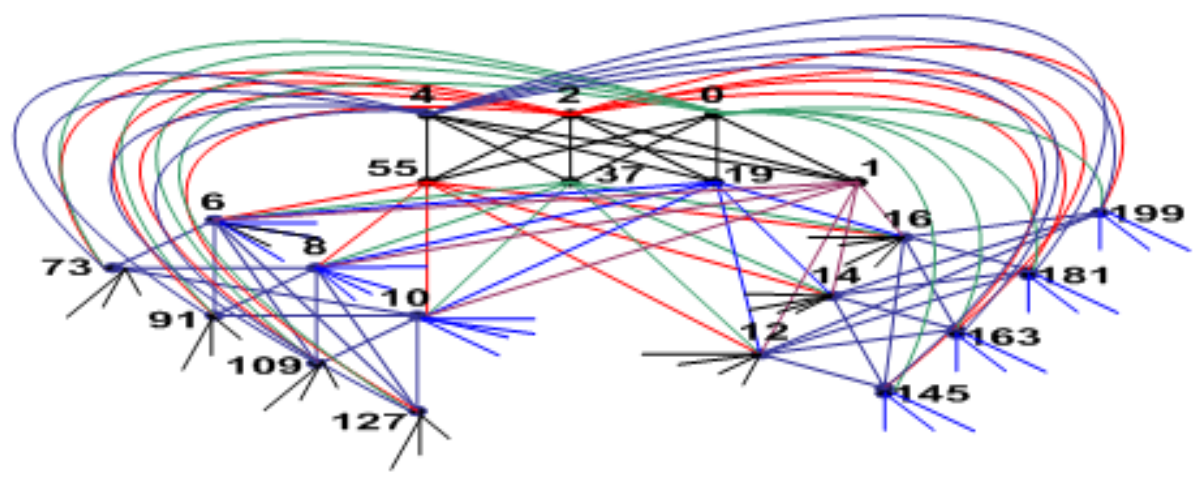

Figure (4): The graph $D_{3}\left(K_{3,4}\right)$ with its odd harmonious labeling.

The following results give odd-harmonious labeling for the "multisplliting" paths and stars. 
International Journal on Applications of Graph Theory in Wireless Ad hoc Networks and Sensor Networks (GRAPH-HOC) Vol.8, No.3/4, December 2016

Theorem 2.6. $\operatorname{Spl}_{m}\left(P_{n}\right)$ is an odd harmonious graph.

Proof. Let $u_{1}^{0}, u_{2}^{0}, u_{3}^{0}, \ldots, u_{n}^{0}$ be the vertices of $P_{n}$ and suppose $u_{1}^{j}, u_{2}^{j}, u_{3}^{j}, \ldots, u_{n}^{j}, \quad 1 \leq j \leq m$ be the $j^{\text {th }}$ vertices corresponding to $u_{1}^{0}, u_{2}^{0}, u_{3}^{0}, \ldots, u_{n}^{0}$, which are added to obtain $\operatorname{Spl}_{m}\left(P_{n}\right)$. Let $\mathrm{G}$ be the graph $\operatorname{Spl}_{m}\left(P_{n}\right)$ described as indicated in Figure(5)

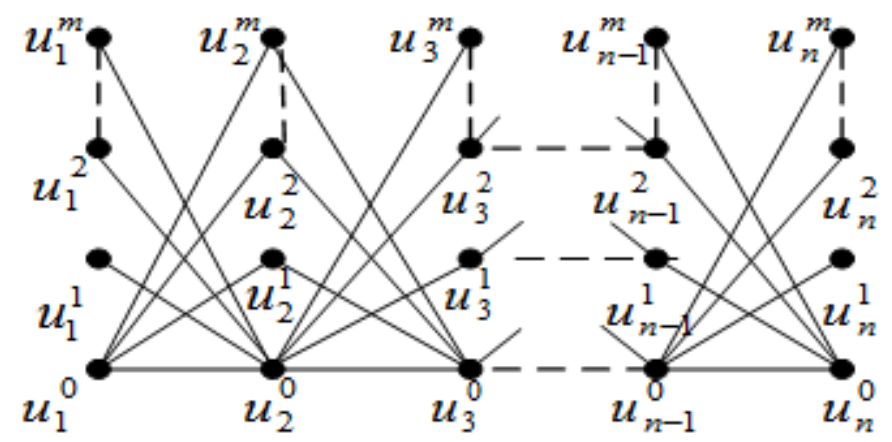

Figure (5)

Then $|V(G)|=n(m+1)$ and $|E(G)|=(n-1)(2 m+1)$. We define

$f: V(G) \rightarrow\{0,1,2, \ldots, 2(n-1)(2 m+1)-1\}$ as follows:

$$
\begin{aligned}
& f\left(u_{i}^{0}\right)=i-1, \quad 1 \leq i \leq n . \\
& f\left(u_{i}^{j}\right)= \begin{cases}4(n-1) j+i-1, & i=1,3,5, \ldots, n \text { or } n-1, \quad 1 \leq j \leq m, \\
2(n-1)(2 j-1)+i-1, & i=2,4,6, \ldots, n-1 \text { or } n, \quad 1 \leq j \leq m .\end{cases}
\end{aligned}
$$

It follows that $f$ admits an odd harmonious labeling for $\operatorname{Spl}_{m}\left(P_{n}\right)$. Hence $\operatorname{Spl}_{m}\left(P_{n}\right)$ is an odd harmonious graph.

Example 2.7. Odd harmonious labeling of the graph $\operatorname{Spl}_{3}\left(P_{7}\right)$ is shown in Figure (6).

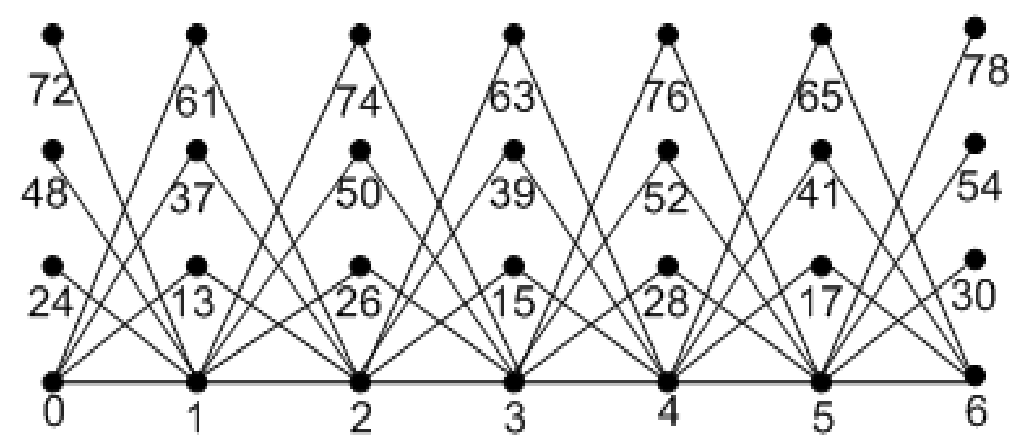

Figure (6): The graph $\operatorname{Spl}_{3}\left(P_{7}\right)$ with its odd harmonious labeling. 
International Journal on Applications of Graph Theory in Wireless Ad hoc Networks and Sensor Networks (GRAPH-HOC) Vol.8, No.3/4, December 2016

Theorem 2.8. $\operatorname{Spl}_{m}\left(K_{l, n}\right)$ is an odd harmonious graph.

Proof. Let $u_{1}, u_{2}, u_{3}, \ldots, u_{n}$ be the pendant vertices and $u_{0}$ be the center of $K_{l, n}$, and $u_{0}^{j}, u_{1}^{j}, u_{2}^{j}, \ldots, u_{n}^{j}, 1 \leq j \leq m$ are the added vertices corresponding to $u_{0}, u_{1}, u_{2}, u_{3}, \ldots, u_{n}$ to obtain $\operatorname{Spl}_{m}\left(K_{l, n}\right)$. Let $G$ be the graph $\operatorname{Spl}_{m}\left(K_{l, n}\right)$. Then $|V(G)|=(n+1)(m+1)$ and $|E(G)|=$ $n(2 m+1)$. We define the vertex labeling function:

$f: V(G) \rightarrow\{0,1,2, \ldots, 2 n(2 m+1)-1\}$ as follows:

$f\left(u_{0}\right)=0$,

$f\left(u_{i}\right)=2 i-1, \quad 1 \leq i \leq n$,

$f\left(u_{0}^{j}\right)=2 n j, \quad 1 \leq j \leq m$,

$f\left(u_{i}^{j}\right)=2[n(m+j)+i]-1, \quad 1 \leq i \leq n, 1 \leq j \leq m$.

It follows that $f$ admits an odd harmonious labeling for $\operatorname{Spl}_{m}\left(K_{l, n}\right)$. Hence $\operatorname{Spl}_{m}\left(K_{l, n}\right)$ is an odd harmonious graph

Example 2.9. An odd harmonious labeling of the graph $\operatorname{Spl}_{3}\left(K_{l, 3}\right)$ is shown in Figure (7).

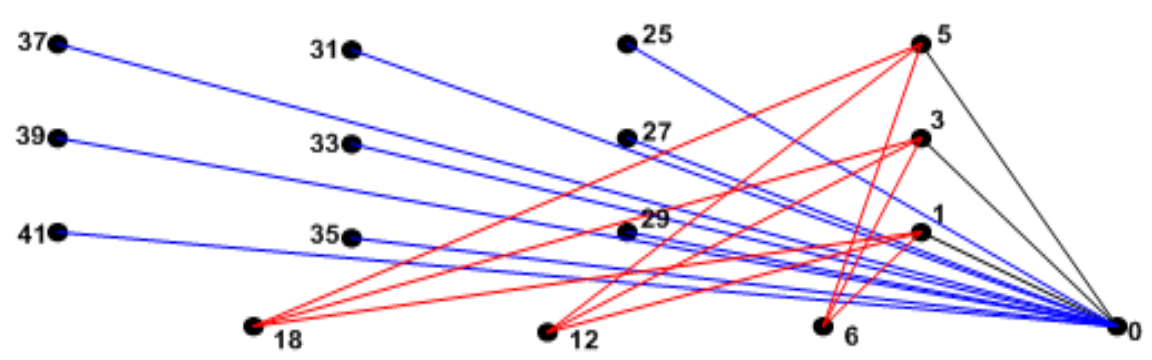

Figure (7) The graph $\operatorname{Spl}_{3}\left(K_{l, 3}\right)$ with its odd harmonious labeling

\section{Remark 2.10.}

In Theorem 2.2, if we take $m=2$ we obtain the known shadow path and when we take $m=2, r=$ 1 in Theorem 2.4 we obtain the known shadow star. Also, in Theorems 2.6, 2.8 if we take $m=1$ we obtain the splitting path and star. These special cases of our results coincide with results of Vaidya and Shah [5] (Theorems 2.1, 2.2, 2.3, 2.4). 
International Journal on Applications of Graph Theory in Wireless Ad hoc Networks and Sensor Networks (GRAPH-HOC) Vol.8, No.3/4, December 2016

\section{SOME ODD HARMONIOUS GRAPHS}

Let $G_{1}$ and $G_{2}$ be two disjoint graphs. The symmetric product $\left(G_{1} \oplus G_{2}\right)$ of $G_{1}$ and $G_{2}$ is the graph having vertex set $V\left(G_{1}\right) \times V\left(G_{2}\right)$ and edge $\operatorname{set}\left\{\left(u_{1}, v_{l}\right)\left(u_{2}, v_{2}\right): u_{1} u_{2} \in E\left(G_{1}\right)\right.$ or $v_{1} v_{2} \in E\left(G_{2}\right)$ but not both\}[4].

Theorem 3.1.

The graphs $P_{n} \oplus \overline{K_{m}}, m, n \geq 2$ are odd-harmonious.

Proof.

Let $P_{n} \oplus \overline{K_{m}}$ be described as indicated in Figure (8):

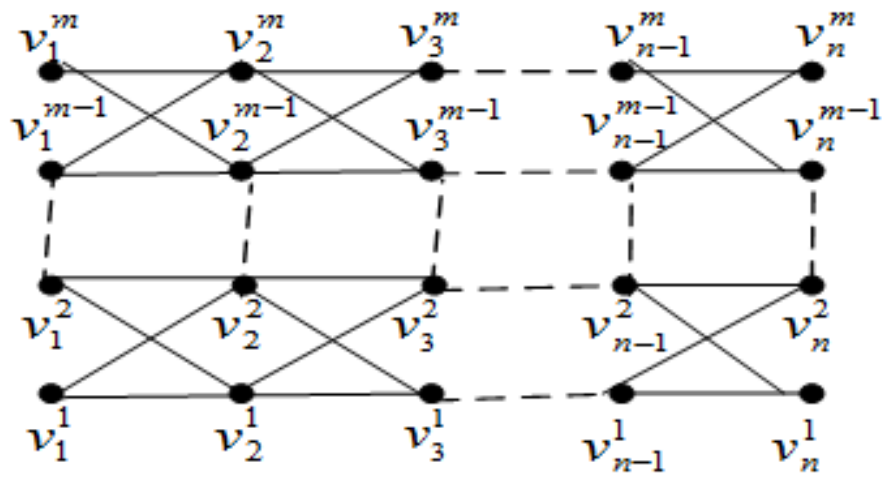

Figure(8)

It is clear that the number of edges of the graph $P_{n} \oplus \overline{K_{m}}$ is $(3 m-2)(n-1)$. We define the labeling function

$f: V\left(P_{n} \oplus \overline{K_{m}}\right) \rightarrow\{0,1,2, \ldots, 2(3 m-2)(n-1)-1\}$

as follows:

$f\left(v_{i}^{j}\right)=\left\{\begin{array}{lll}(3 m-2)(i-1)+4(j-1), & i=1,3,5, \ldots n \text { or } n-1, & j=1,2,3, \ldots m \\ (3 m-2) i-(6 m-5)+2(j-1), & i=2,4,6, \ldots n-1 \text { or } n-1, \quad j=1,2,3, \ldots m\end{array}\right.$

It follows that $f$ admits an odd harmonious labeling for $P_{n} \oplus \overline{K_{m}}$. Hence $P_{n} \oplus \overline{K_{m}}$ is an odd harmonious graph. 
International Journal on Applications of Graph Theory in Wireless Ad hoc Networks and Sensor Networks (GRAPH-HOC) Vol.8, No.3/4, December 2016

Example 3.2. An odd harmonious labeling of the graph $P_{6} \oplus \overline{K_{3}}$ is shown in Figure (9).

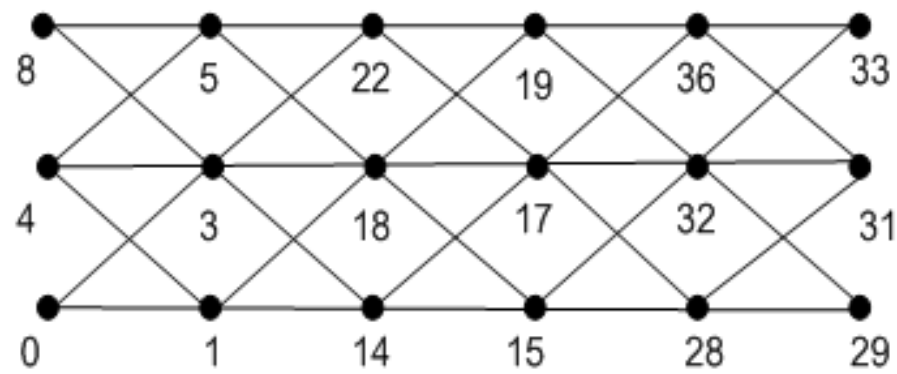

Figure (9): The graph $P_{6} \oplus \overline{K_{3}}$ with its odd harmonious labeling.

\section{Theorem 3.3.}

The graphs $\operatorname{Spl}_{m}\left(P_{n} \oplus \overline{K_{2}}\right), m, n \geq 2$ are odd-harmonious.

Proof. Let $u_{1}, u_{2}, u_{3}, \ldots, u_{n} ; v_{1}, v_{2}, v_{3}, \ldots, v_{n}$ be the vertices of the graph $P_{n} \oplus \overline{K_{2}}$ and suppose $u_{1}^{j}, u_{2}^{j}, u_{3}^{j}, \ldots, u_{n}^{j}, 1 \leq j \leq m$ be the $j^{\text {th }}$ vertices corresponding to $u_{1}, u_{2}, u_{3}, \ldots, u_{n}$ and $v_{1}^{j}, v_{2}^{j}, v_{3}^{j}, \ldots, v_{n}^{j}, 1 \leq j \leq m$ be the $j^{\text {th }}$ vertices corresponding to $v_{1}, v_{2}, v_{3}, \ldots, v_{n}$ which are added to obtain $\operatorname{Spl}_{m}\left(P_{n} \oplus \overline{K_{2}}\right)$. The graph $\operatorname{Spl}_{m}\left(P_{n} \oplus \overline{K_{2}}\right)$ is described as indicated in Figure (10)

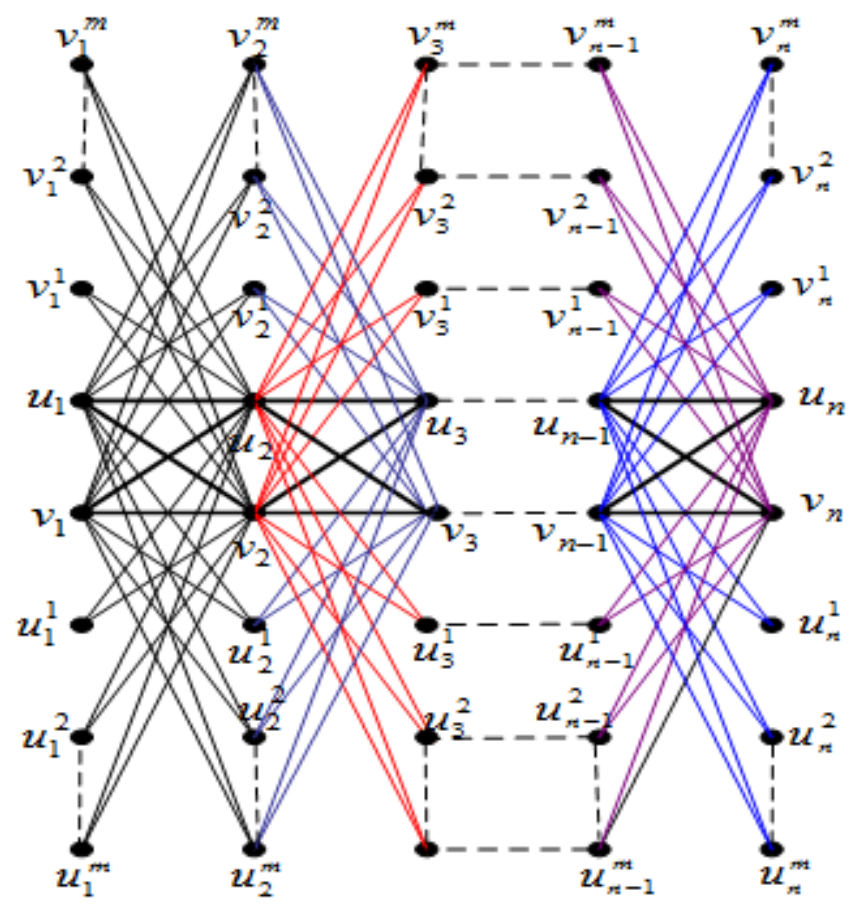

Figure (10) 
International Journal on Applications of Graph Theory in Wireless Ad hoc Networks and Sensor Networks (GRAPH-HOC) Vol.8, No.3/4, December 2016

Then the number of edges of the graph $\operatorname{Spl}_{m}\left(P_{n} \oplus \overline{K_{2}}\right)=4(2 m+1)(n-1)$. We define:

$f: V\left(\operatorname{Spl}_{m}\left(P_{n} \oplus \overline{K_{2}}\right)\right) \rightarrow\{0,1,2, \ldots 8(2 m+1)(n-1)-1\}$.

First, we consider the labeling for the graph $P_{n} \oplus \overline{K_{2}}$ as follows:

$$
\begin{aligned}
& f\left(u_{i}\right)= \begin{cases}4(m+1)+4(2 m+1)(i-1), & i=1,3,5, \ldots n \text { or } n-1 \\
4(2 m+1)(i-2)+3, & i=2,4,6, \ldots n-1 \text { or } n .\end{cases} \\
& f\left(v_{i}\right)= \begin{cases}4 m+4(2 m+1)(i-1), & i=1,3,5, \ldots n \text { or } n-1 \\
4(2 m+1)(i-2)+1, & i=2,4,6, \ldots n-1 \text { or } n .\end{cases}
\end{aligned}
$$

For labeling the added vertices $u_{i}^{j}, v_{i}^{j}, 1 \leq i \leq n, 1 \leq j \leq m$ we consider the following three cases:

\section{Case (i)}

if $i$ is odd, $1 \leq i \leq n$ we have the following labeling, for each $1 \leq j \leq m$

$$
\begin{aligned}
& f\left(u_{i}^{j}\right)=4(m-j)+4(2 m+1)(i-1) \\
& f\left(v_{i}^{j}\right)=4(m+j+1)+4(2 m+1)(i-1)
\end{aligned}
$$

\section{Case (ii)}

if $i$ even, $1 \leq i \leq n$ and $m \equiv 1(\bmod 2), 1 \leq j \leq m$ we have the following labeling:

$$
\begin{aligned}
& f\left(u_{i}^{j}\right)= \begin{cases}4(2 m+2-j)+4(2 m+1)(i-2)+1, & j=1,3,5, \ldots m \\
4(2 m+2-j)+4(2 m+1)(i-2)-1, & j=2,4,6, \ldots m-1\end{cases} \\
& f\left(v_{i}^{j}\right)= \begin{cases}4(2 m+1+j)+4(2 m+1)(i-2)-1, & j=1,3,5, \ldots m \\
4(2 m+1+j)+4(2 m+1)(i-2)+1, & j=2,4,6, \ldots m-1\end{cases}
\end{aligned}
$$


International Journal on Applications of Graph Theory in Wireless Ad hoc Networks and Sensor Networks (GRAPH-HOC) Vol.8, No.3/4, December 2016

\section{Case (iii)}

if $i$ even, $1 \leq i \leq n$ and $m \equiv 0(\bmod 2), 1 \leq j \leq m$ we have the following labeling:

$$
\begin{aligned}
& f\left(u_{i}^{j}\right)= \begin{cases}4(2 m+2-j)+4(2 m+1)(i-2)-1, & j=1,3,5, \ldots m-1 \\
4(2 m+2-j)+4(2 m+1)(i-2)+1, & j=2,4,6, \ldots m\end{cases} \\
& f\left(v_{i}^{j}\right)= \begin{cases}4(2 m+1+j)+4(2 m+1)(i-2)+1, & j=1,3,5, \ldots m-1 \\
4(2 m+1+j)+4(2 m+1)(i-2)-1, & j=2,4,6, \ldots m\end{cases}
\end{aligned}
$$

It follows that $f$ admits an odd harmonious labeling for $\operatorname{Spl}_{m}\left(P_{n} \oplus \overline{K_{2}}\right)$. Hence $\operatorname{Spl}_{m}\left(P_{n} \oplus \overline{K_{2}}\right)$ is an odd harmonious graph.

Example 3.4. Odd harmonious labelings of graphs $\operatorname{Spl}_{2} \quad\left(\begin{array}{llll}P_{5} & \oplus & \overline{K_{2}}\end{array}\right)$ and $\mathrm{Spl}_{3}\left(P_{5} \oplus \overline{K_{2}}\right)$ are shown in Figure (11a) and Figure (11b) respectively.

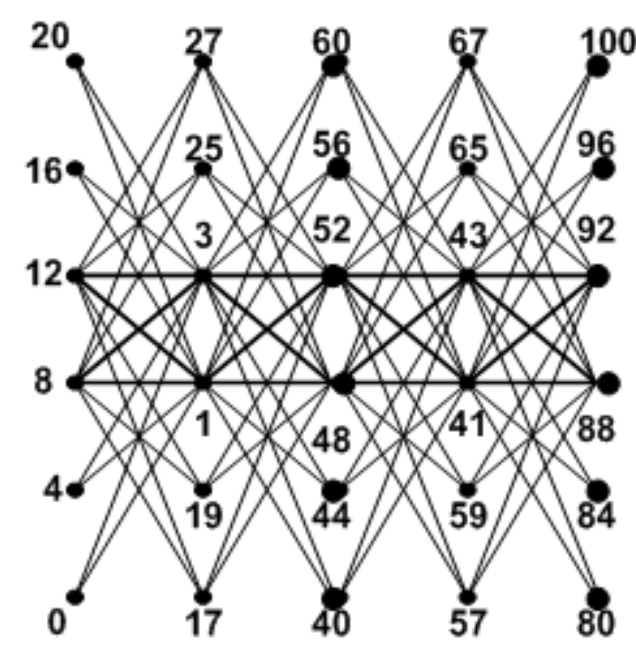

Figure (11a) $m \equiv 0(\bmod 2)$,

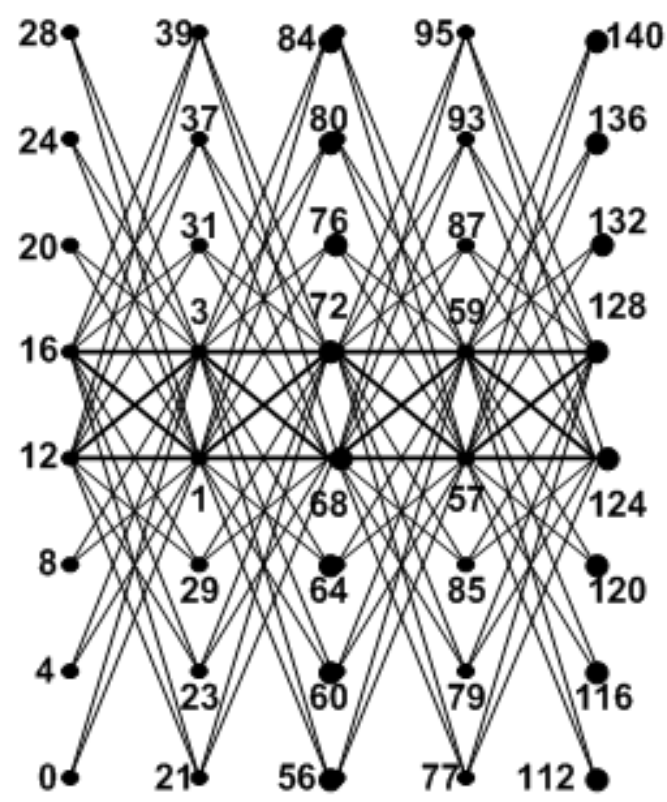

Figure $(11 \mathrm{~b}), m \equiv 1(\bmod 2)$

Figure (11a), Figure (11b): The graphs $\operatorname{Spl}_{2}\left(P_{5} \oplus \overline{K_{2}}\right)$ and $\operatorname{Spl}_{3}\left(P_{5} \oplus \overline{K_{2}}\right)$ with their odd harmonious labeling. 
International Journal on Applications of Graph Theory in Wireless Ad hoc Networks and Sensor Networks (GRAPH-HOC) Vol.8, No.3/4, December 2016

Let $G_{1}$ and $G_{2}$ be two disjoint graphs. The conjunction $\left(G_{1} \wedge G_{2}\right)$ of $G_{1}$ and $G_{2}$ is the graph having vertex set $V\left(G_{1}\right) \times V\left(G_{2}\right)$ and edge $\operatorname{set}\left\{\left(u_{1}, v_{l}\right)\left(u_{2}, v_{2}\right): u_{1} u_{2} \in E\left(G_{1}\right)\right.$ and $\left.v_{1} v_{2} \in E\left(G_{2}\right)\right\}[4]$.

\section{Theorem 3.5.}

The graphs $\operatorname{Spl}_{m}\left(P_{2} \wedge S_{n}\right), m, n \geq 2$ are odd harmonious.

\section{Proof.}

Let $u_{0}^{0}, u_{1}^{0}, u_{2}^{0}, \ldots, u_{n}^{0} ; v_{0}^{0}, v_{1}^{0}, v_{2}^{0}, \ldots, v_{n}^{0}$ be the vertices of the graph $P_{2} \wedge S_{n}$ and suppose $u_{0}^{j}, u_{1}^{j}, u_{2}^{j}, u_{3}^{j}, \ldots, u_{n}^{j}, \quad 1 \leq j \leq m$ be the $j^{\text {th }}$ vertices corresponding to $u_{0}^{0}, u_{1}^{0}, u_{2}^{0}, \ldots, u_{n}^{0}$ and $v_{0}^{j}, v_{1}^{j}, v_{2}^{j}, v_{3}^{j}, \ldots, v_{n}^{j}, 1 \leq j \leq m$ be the $j^{\text {th }}$ vertices corresponding to $v_{0}^{0}, v_{1}^{0}, v_{2}^{0}, \ldots, v_{n}^{0}$ which are added to obtain $\operatorname{Spl}_{m}\left(P_{2} \wedge S_{n}\right)$. The graph $\operatorname{Spl}_{m}\left(P_{2} \wedge S_{n}\right)$ is described as indicated in figure (12)

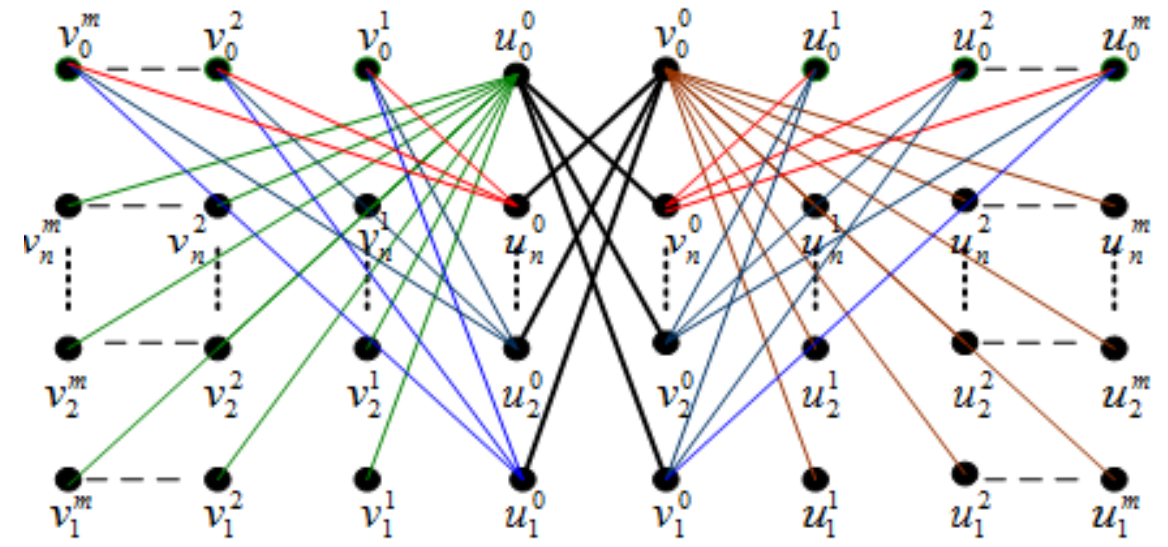

Figure (12)

It is clear that the number of edges of the graph $\operatorname{Spl}_{m}\left(P_{2} \wedge S_{n}\right)$ is $2 n(2 m+1)$. Now we define the labeling function

$f: V\left(S p l_{m}\left(P_{2} \wedge S_{n}\right)\right) \rightarrow\{0,1,2, \ldots, 4 n(2 m+1)-1\}$,

as follows:

$$
\begin{array}{ll}
f\left(u_{0}^{j}\right)=(2 m+2) n-1+2 j, & 0 \leq j \leq m \\
f\left(v_{0}^{j}\right)=(2 m+1)-2 j, & 0 \leq j \leq m \\
f\left(v_{i}^{0}\right)=2 i(m+1)-2 m, & 1 \leq i \leq n \\
f\left(u_{i}^{0}\right)=2(m+1)(i-1), & 1 \leq i \leq n
\end{array}
$$

Now we label the remaining vertices $u_{i}^{j}, v_{i}^{j}, 1 \leq i \leq n, 1 \leq j \leq m$ as follows: 
International Journal on Applications of Graph Theory in Wireless Ad hoc Networks and Sensor Networks (GRAPH-HOC) Vol.8, No.3/4, December 2016

For the vertices $v_{i}^{j}$ : we put the labeled vertices $v_{i}^{1}, v_{i}^{2}, v_{i}^{3}, \ldots, v_{i}^{m}, 1 \leq i \leq n$, ordered from bottom to top in columns as in Example 3.6, the labels form an arithmetic progression whose basis is 2 and whose first term is $2[(m+1) n+1]$.

Similarly for $u_{i}^{j}$ : we put the labeled vertices $u_{i}^{1}, u_{i}^{2}, u_{i}^{3}, \ldots, u_{i}^{m}, 1 \leq i \leq n$, ordered from bottom to top in columns as in Example 3.6, the labels form an arithmetic progression whose basis is 2 and whose first term is $2[(3 n-1) m+2 n]$.

It follows that $f$ admits an odd harmonious labeling for $\operatorname{Spl}_{m}\left(P_{2} \wedge S_{n}\right)$. Hence $\operatorname{Spl}_{m}\left(P_{2} \wedge S_{n}\right)$ is an odd harmonious graph.

Example 3.6. An odd harmonious labeling of the graph $\operatorname{Spl}_{3}\left(P_{2} \wedge S_{4}\right)$ is shown in Figure (13).

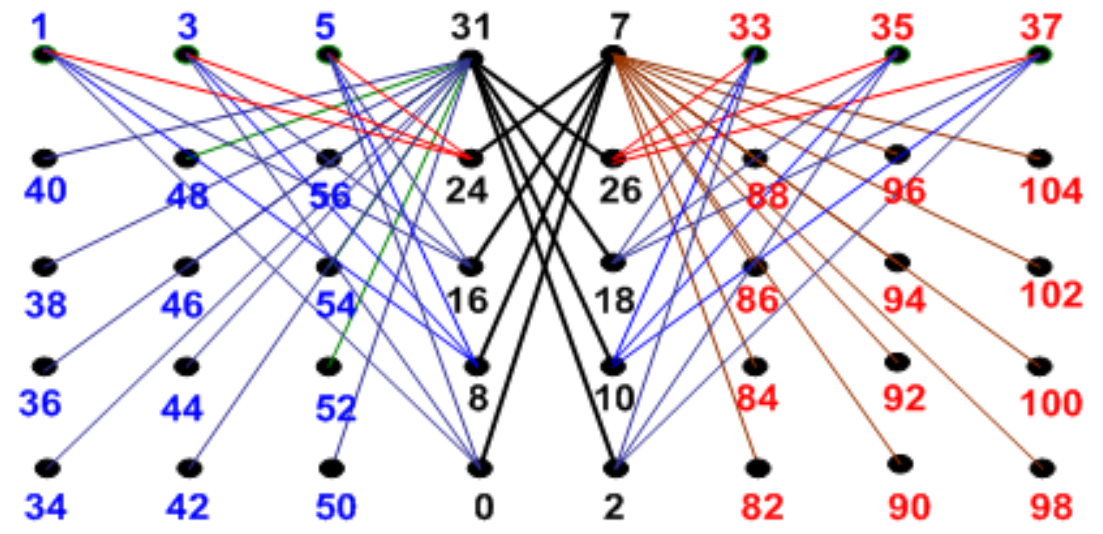

Figure (13): The graph $\operatorname{Spl}_{3}\left(P_{2} \wedge S_{4}\right)$ with its odd harmonious labeling

\section{Corollary 3.7.}

The following known graphs are odd harmonious:

1. $\operatorname{Spl}\left(S_{n} \wedge P_{2}\right) \quad, \quad n \geq 2$

2. $\operatorname{Spl}\left(P_{n} \oplus \overline{K_{2}}\right), n \geq 2$

\section{Theorem 3.8.}

The graphs $\operatorname{Spl}\left(\mathrm{K}_{\mathrm{m}, \mathrm{n}}\right), \mathrm{m}, \mathrm{n} \geq 2$ are odd harmonious.

Proof.

Let $V\left(K_{m, n}\right)=\left\{u_{1}, u_{2}, u_{3}, \ldots, u_{m} ; v_{l}, v_{2}, v_{3}, \ldots, v_{n}\right\}, m, n \geq 2$. It is clear that the number of edges of the graph $\operatorname{Spl}\left(K_{m, n}\right)$ is $3 m n$. The graph is indicated in Figure (14): 
International Journal on Applications of Graph Theory in Wireless Ad hoc Networks and Sensor Networks (GRAPH-HOC) Vol.8, No.3/4, December 2016

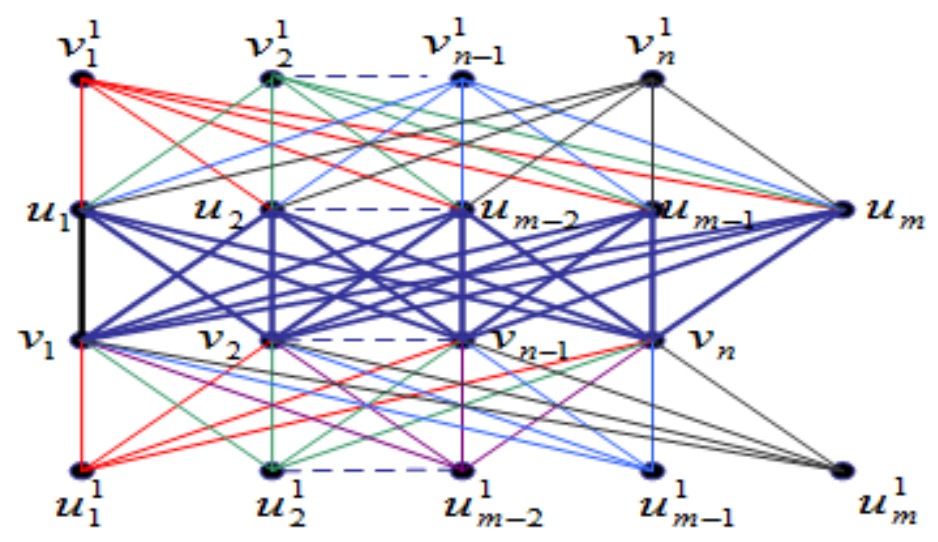

Figure (14)

We define the labeling function

$f: V\left(\operatorname{Spl}\left(K_{m, n}\right)\right) \rightarrow\{0,1,2, \ldots, 6 m n-1\}$ as follows:

$f\left(v_{i}\right)=4 \mathrm{i}-3 \quad, \quad 1 \leq i \leq m$

$f\left(u_{i}\right)=6 n(i-1), \quad 1 \leq i \leq n$

$f\left(v_{\mathrm{i}}^{1}\right)=4 n+2 i-1 \quad, \quad 1 \leq i \leq m$

$f\left(u_{\mathrm{i}}^{1}\right)=2+6 n(i-1), \quad 1 \leq i \leq n$.

It follows that $f$ admits an odd harmonious labeling for $\operatorname{Spl}\left(K_{m, n}\right)$. Hence $\operatorname{Spl}\left(K_{m, n}\right)$ is an odd harmonious graph.

Example 3.9. Odd harmonious labeling of graph $\operatorname{Spl}\left(K_{3,4}\right)$ is shown in Figure (15).

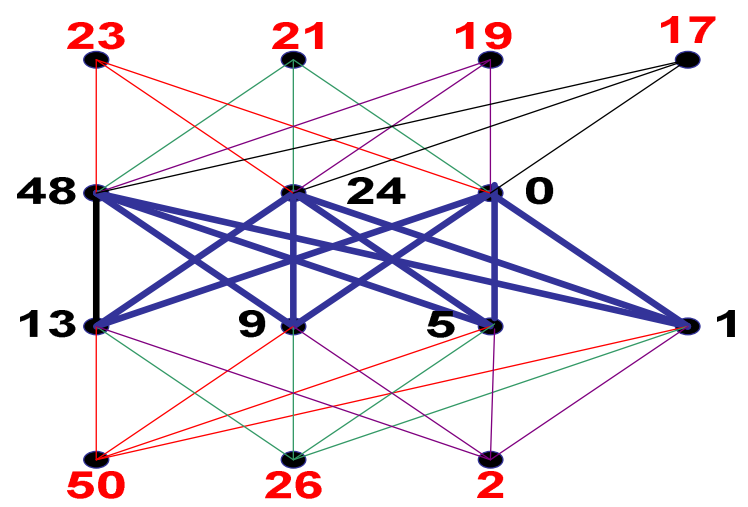

Figure (15): The graph $\operatorname{Spl}\left(K_{3,4}\right)$ with its odd harmonious labeling 
International Journal on Applications of Graph Theory in Wireless Ad hoc Networks and Sensor Networks

(GRAPH-HOC) Vol.8, No.3/4, December 2016

\section{REFERENCES}

[1] M. E. Abdel-Aal. New Classes of Odd Graceful Graphs, J. (GRAPH-HOC) Vol. 5, No. 2 (2013), 112.

[2] J. A. Gallian. A Dynamic Survey of Graph Labeling, Electronic J. Combin. Fiftteenth edition, (2012).

[3] R. L. Graham, N. J. A. Sloane. On additive bases and harmonious graphs, SIAM J. Algebr. Disc. Math., 4 (1980), 382-404.

[4] F. Harary. GpaphTheory, Addison-Wesley, Reading MA (1969).

[5] Z. Liang, Z. Bai. On the odd harmonious graphs with applications, J. Appl.Math. Comput., 29 (2009), 105-116.

[6] S.K. Vaidya, N. H. Shah. Some new odd harmonious graphs, International Journal of Mathematics and Soft Computing, 1 (2011), 9-16.

\section{AUTHOR}

Mohamed Elsayed Abdel-Aal received the B.Sc. (Mathematics) the M.Sc.(Pure Mathematics-Abstract Algebra) degree from Benha University, Benha, Egypt in 1999, 2005 respectively. Also, he received Ph.D. (Pure Mathematics) degree from Faculty of Mathematics, Tajik National University, Tajikistan, in 2011. He is a University lecturer of Pure Mathematics with the Benha University, Faculty of Science, Department of Pure Mathematics. His current research is Ordinary -partial differential equations, Graph Theory and Abstract Algebra.

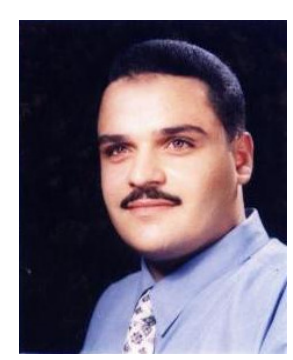

